\title{
AVALIAÇÃO DA PÓS-GRADUAÇÃO DA ÁREA DE QUÍMICA NA CAPES E A INTERNACIONALIZAÇÃO DAS REVISTAS DA SOCIEDADE BRASILEIRA DE QUÍMICA: JOURNAL OF THE BRAZILIAN CHEMICAL SOCIETY E QUÍMICA NOVA
}

\author{
Angelo C. Pinto e Andréa S. Cunha* \\ Instituto de Química, Universidade Federal do Rio de Janeiro, Centro de Tecnologia, Cidade Universitária, 21949-900 Rio de \\ Janeiro - RJ, Brasil \\ Recebido em 6/11/08

\begin{abstract}
EVALUATION OF THE CHEMISTRY GRADUATE COURSES BY CAPES (COORDENAÇÃO DE PESSOAL DE ENSINO SUPERIOR) AND THE INTERNATIONALIZATION OF THE JOURNAL OF THE BRAZILIAN CHEMICAL SOCIETY AND QUIMICA NOVA. This paper discusses of the use of the Impact Factor in the evaluation of chemistry graduate courses by CAPES and the internationalization of the Journal of the Brazilian Chemical Society and Química Nova.
\end{abstract}

Keywords: Qualis; impact factor; CAPES; J. Braz. Chem. Soc.; Quim. Nova.

\section{INTRODUÇÃO}

Passados quase 10 anos desde que o artigo "Fator de Impacto de Revistas Científicas: Qual o significado Deste Parâmetro?" ${ }^{1}$ foi publicado na Química Nova, apesar dos prós e dos contras ${ }^{2-4}$ sobre a aplicação do Fator de Impacto como critério de identificação de qualidade de produção científica, ${ }^{5}$ o CTC da CAPES determinou que as coordenações de áreas usem este indicador para avaliação e conceituação dos Programas de Pós-Graduação.

Em 2008, 28 periódicos "brasileiros" estavam listados no banco de dados Web of Science (Quadro 1), 7 a mais do que no ano anterior. Em 2002 eram apenas 16 as revistas brasileiras neste banco de dados. A indexação ao Web of Science foi sempre uma das cobranças dos presidentes do CNPq e da CAPES aos editores dos periódicos brasileiros.

O emprego de indicadores para qualificar tanto periódicos científicos quanto o trabalho de pesquisadores é muito salutar e necessário, e deve fazer parte da rotina dos comitês de agências de fomento nas suas avaliações. Apesar de todas as críticas que são feitas ao Fator de Impacto, este indicador deve continuar sendo usado pelos comitês, mas não como critério absoluto de qualidade. É muito importante que os comitês conheçam as inconsistências dos indicadores que empregam nas suas avaliações.

A CAPES avançou com seu sistema de avaliação quando criou a base de dados Qualis. ${ }^{6}$ Esta base de dados reúne apenas os títulos de periódicos utilizados pelos Programas de Pós-Graduação para a divulgação da produção intelectual de seus docentes e alunos, e é usada pela CAPES como um dos instrumentos do processo de avaliação do sistema nacional de Pós-Graduação. O Qualis que foi utilizado no triênio 2004-2006 classificou os periódicos de acordo com o âmbito de sua circulação como em Local, Nacional e Internacional, e em $\mathbf{A}, \mathbf{B}$ ou $\mathbf{C}$ quanto à qualidade. Apesar do Fator de Impacto ser usado para a classificação e estratificação dos periódicos no Qualis, as coordenações podem usar outros critérios para classificarem as revistas de suas áreas.

Os representantes das áreas de Ciências Biológicas II e de Química quando em algumas avaliações passadas organizaram o Qualis de suas áreas com base nos fatores de impacto de um grande número de revistas publicado pelo Journal Citation Reports, decidiram para

*e-mail: andreacunha@iq.ufrj.br

Quadro 1. Os periódicos brasileiros listados, em 2008, Web of Science

Periódicos Indexados no Web of Science

1 Anais da Academia Brasileira de Ciências

2 Arquivo Brasileiro de Medicina Veterinária e Zootecnia

3 Arquivos de Neuro-Psiquiatria

4 Bulletin of the Brazilian Mathematical Society

5 Brazilian Archives of Biology and Technology

6 Brazilian Journal of Chemical Engineering

7 Brazilian Journal of Medical and Biological Research

8 Brazilian Journal of Microbiology

9 Brazilian Journal of Oceanography

10 Brazilian Journal of Physics

11 Ciência e Tecnologia de Alimentos

12 Ciência Florestal

13 Genetics and Molecular Biology

14 Iheringia. Série Zoologia

15 Journal of The Brazilian Chemical Society

16 Journal of Venomous Animals and Toxins

17 Memórias do Instituto Oswaldo Cruz

18 Neotropical Entomology

19 Neotropical Ichthyology

20 Pesquisa Agropecuária Brasileira

21 Pesquisa Veterinária Brasileira

22 Química Nova

23 Revista Brasileira de Ciência do Solo

24 Revista Brasileira de Entomologia

25 Revista Brasileira de Zoologia

26 Revista Brasileira de Zootecnia

27 Revista da Sociedade Brasileira de Medicina Tropical

28 Scientia Agraria 
incentivar o crescimento das revistas Brazilian Journal of Medical and Biological Research e o Journal of the Brazilian Chemical Society, respectivamente, classificá-las como Qualis A e internacional $\mathbf{A}$, apesar dos fatores de impacto destes periódicos serem inferiores aos periódicos classificados nestes estratos. A coordenação da área de Química foi ainda mais além na organização do Qualis no triênio 2004-2006, e classificou tanto a Química Nova como os Anais da Academia Brasileira de Ciências como internacional B. ${ }^{7}$

Recentemente, a revista FAPESP publicou com o título " $O$ fôlego na Berlinda", ${ }^{8}$ o resultado de dois rankings de produção científica mundial que foram divulgados em julho de 2008 sobre o desempenho acadêmico do Brasil em 2007. A base de dados Thomson Scientific mostrou que o Brasil foi responsável, em 2007, por 2,02\% e a base de dados Scopus, comercializada pela editora Elsevier, por $1,75 \%$ do total da produção científica mundial, no mesmo ano. Mas, ambos os bancos de dados colocam o Brasil na $15^{\mathrm{a}}$ posição no ranking mundial de produção científica, à frente de países com maior tradição de pesquisa e formação de recursos humanos. A discrepância nos percentuais é devido ao número de periódicos indexados a cada um destes bancos de dados. Enquanto a ferramenta Web of Science, da Thomson Scientific, cobre cerca de 10 mil periódicos, a ferramenta SCImago, da Scopus, cobre 15 mil. Mais curioso do que a diferença de percentuais, é o fato da CAPES usar o Web of Science para instrumentalizar o Qualis, e o CNPq o Scopus para a avaliação dos pesquisadores. Esta, entretanto, é uma outra história, o importante é que aumenta, a cada ano, o número de periódicos brasileiros indexados nas duas principais bases de dados internacionais. Este aumento demonstra o grau de maturidade atingido pela comunidade científica brasileira e a importância que uma parcela considerável dos editores dão à internacionalização de suas revistas.

\section{INTERNACIONALIZAÇÃO DA PRODUÇÃO CIENTÍFICA BRASILEIRA}

O critério que sempre é usado quando se faz menção à internacionalização da produção científica brasileira corresponde aos artigos produzidos no País que estão no portal ISI Web of Knowledge, que inclui os acessos ao Web of Science e ao Journal Citation Reports. Mais recentemente vem ganhando espaço no País no meio acadêmico a base Scopus. O conceito de internacionalização pode ser ampliado, por exemplo, para pesquisadores do exterior que publicam seus artigos em revistas brasileiras. Talvez seja este atualmente o critério mais importante para a definição de internacionalização da produção científica brasileira. Este é um dos motivos pelos quais os atuais editores do Journal of the Brazilian Chemical Society buscam a sua consolidação como a principal revista de Química das nações em desenvolvimento e que o JBCS atraia, cada vez mais, mais artigos de pesquisadores do exterior.

Desde 2006, o número de manuscritos do exterior submetidos ao $J B C S$ é maior do que os submetidos por autores que realizam seus trabalhos no Brasil (Quadro 2).

Quando se faz a análise dos artigos publicados no $J B C S$, em 2007, se constata que dos 206 artigos publicados nesse ano na re-

Quadro 2. Comparação do número de manuscritos submetidos ao JBCS em 2006, 2007 e 2008

\begin{tabular}{|lcc|}
\hline & Exterior & Brasil \\
\hline 2006 & 391 & 322 \\
2007 & 394 & 313 \\
2008 (até 31/10) & 393 & 256 \\
\hline
\end{tabular}

vista, 58 foram de pesquisadores do exterior (Tabela 1). O número de submissões de manuscritos do exterior é maior do que o número de submissões de manuscritos do Brasil. Na Tabela 1 não foram relacionados os artigos publicados por pesquisadores estrangeiros em co-autoria com pesquisadores brasileiros.

O que chama atenção na Tabela 1 é o número de artigos oriundos do Irã, da China e da Índia, que pode ser melhor observado na Figura 1. No caso do Irã, uma das possíveis explicações pode ser o estremecimento das relações diplomáticas do governo americano com o de Teerã, dada a insistência do governo iraniano em prosseguir com o seu programa nuclear paralelo.

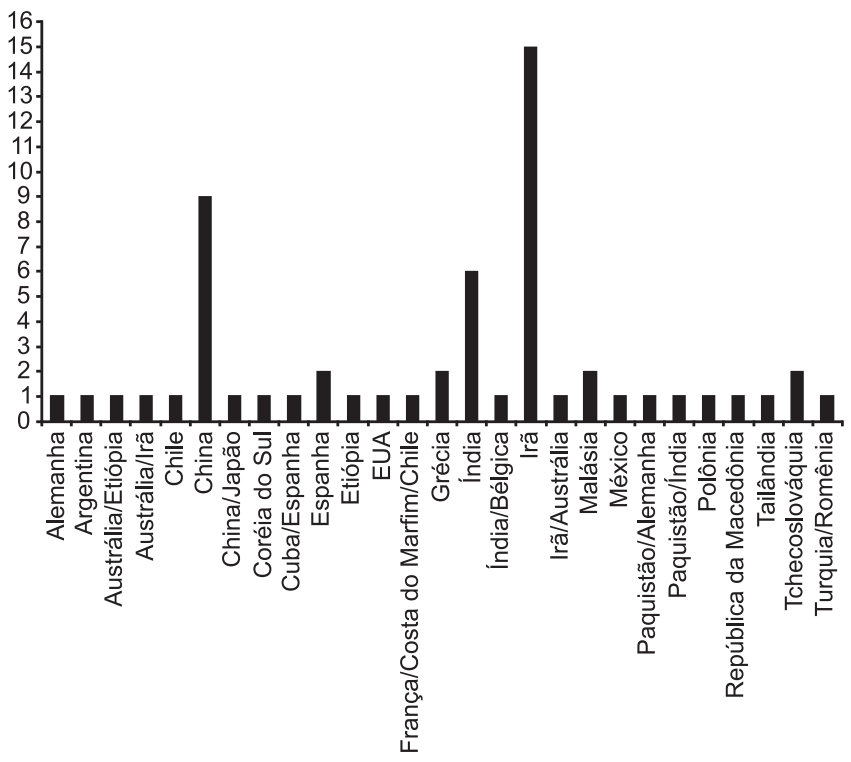

Figura 1. Publicações por país, em 2007, no J. Braz. Chem. Soc.

No caso das publicações da China e da Índia, estas se revestem de grande importância porque estas duas nações ocuparam diariamente, nos últimos três anos, o noticiário internacional como exemplo de economias pujantes, e ciência e comércio internacional caminham de mãos dadas.

Não há dúvida que a economia americana ainda é a mais importante de todo o mundo, sendo responsável por cerca de 21 e $24 \%$ da economia mundial. Mas, é bom lembrar que logo após a Segunda Guerra Mundial a economia americana representava cerca de $50 \%$ da economia mundial. Do mesmo modo que a economia, a produção científica dos EUA cresce em menor proporção do que a de outros países. Aumentar a produção científica brasileira é um dos passos necessários para aumentar a participação do Brasil no comércio mundial. A atração de empresas de alta tecnologia para o Brasil exige, antes de tudo, uma mão de obra científica altamente qualificada. Revistas científicas consolidadas e de qualidade são bons cartões de visita.

A revista Química Nova é a terceira revista com o maior Fator de Impacto no Journal Citation Reports que não é publicada em inglês. Apesar de publicada em sua maior parte em língua portuguesa, dos 342 artigos publicados em 2007 na Química Nova, 16 foram de pesquisadores do exterior (Tabela 2).

Não foram listados na Tabela 2 artigos de pesquisadores do exterior publicados em co-autoria com pesquisadores brasileiros. Devido a sua qualidade e penetração nos países de língua portuguesa e espanhola, a tendência é que Química Nova atraia cada vez mais manuscritos da América Latina e Central, e da Península Ibérica. A Figura 2 apresenta a distribuição das publicações em Química Nova, em 2007, por país. 
Tabela 1. Artigos do exterior publicados em 2007 no J. Braz. Chem. Soc.

\begin{tabular}{|c|c|c|}
\hline Título & Autores & País de origem \\
\hline $\begin{array}{l}\text { A Comparative Evaluation on the Oxidative Approaches for Extraction of Humic } \\
\text { Acids from Low Rank Coal of Mukah, Sarawak }\end{array}$ & Fong, S. S. e cols ${ }^{9}$ & Malásia \\
\hline $\begin{array}{l}\text { Reuse of Nitric Acid in the Oxidative Pretreatment Step for Preparation of Humic } \\
\text { Acids from Low Rank Coal of Mukah, Sarawak }\end{array}$ & Fong, S. S. e cols ${ }^{10}$ & Malásia \\
\hline $\begin{array}{l}\text { A Facile Spectrophotometric Method for the Determination of Hypochlorite using } \\
\text { Rhodamine B }\end{array}$ & Pasha, C. e Narayana, B. ${ }^{11}$ & Índia \\
\hline $\begin{array}{l}\text { Fabrication of a New Samarium(III) Ion-Selective Electrode Based on 3-\{[2-Oxo- } \\
1(2 H) \text {-acenaphthylenyliden]amino }\} \text {-2-thioxo-1,3-thiazolidin-4-one }\end{array}$ & Zamani, H. A. e cols. ${ }^{12}$ & Irã \\
\hline $\begin{array}{l}\text { Novel Method for Determination of Trace Amounts of Citalopram in Tablets } \\
\text { by Fast Fourier Continuous Cyclic Voltammetry at Au Microelectrode in Flowing } \\
\text { Solutions }\end{array}$ & Norouzi, P. e cols. ${ }^{13}$ & Irã \\
\hline $\begin{array}{l}\text { Mono and Dibromo-5,5-diethylbarbituric Acids for Cleavage of Trimethylsilyl } \\
\text { Ethers }\end{array}$ & Khazaei, A. e cols. ${ }^{14}$ & Irã \\
\hline $\begin{array}{l}\text { Spectrophotometric Studies on the Protonation and Nickel Complexation Equilibria } \\
\text { of 4-(2-Pyridylazo) Resorcinol using Global Analysis in Aqueous Solution }\end{array}$ & Ghasemi, J. e cols. ${ }^{15}$ & Irã/Austrália \\
\hline$p$-Nitrobenzoic Acid Promoted Synthesis of 1,5-enzodiazepine Derivatives & Varala, R. e cols. ${ }^{16}$ & Índia \\
\hline Benign Approaches for the Microwave-assisted Synthesis of Quinoxalines & Mohsenzadeh, F. e cols. ${ }^{17}$ & Irã \\
\hline $\begin{array}{l}\text { Hydride Generation-in situ Trapping-flame Atomic Absorption Spectrometry } \\
\text { Hybridization for Indium and Thallium Determination }\end{array}$ & $\begin{array}{l}\text { Matusiewicz, H. e } \\
\text { Krawczyk, M. }\end{array}$ & Polônia \\
\hline $\begin{array}{l}\text { Synthesis of Novel PETT Analogues: 3,4-Dimethoxy Phenyl Ethyl 1,3,5-Triazinyl } \\
\text { Thiourea Derivatives and their Antibacterial and Anti-HIV Studies }\end{array}$ & Patel, R. B. e cols. ${ }^{19}$ & Índia/Bélgica \\
\hline $\begin{array}{l}\text { A Simple Colorimetric Method for the Determination of Carbofuran and its Application } \\
\text { in Environmental and Biological Samples }\end{array}$ & Tamrakar, U. e cols. ${ }^{20}$ & Índia \\
\hline $\begin{array}{l}\text { Application of Pyridine-2-carbaldehyde-2-(4-methyl-1,3-benzo thiazol-2-yl)- } \\
\text { hydrazone as a Neutral Ionophore in the Construction of a Novel Er(III) Sensor }\end{array}$ & Ganjali, M. R. e cols. ${ }^{21}$ & Irã \\
\hline $\begin{array}{l}\text { Potentiometric Study of Cetylpyridinium Chloride Cooperative Binding to Anionic } \\
\text { Azo-Dyes }\end{array}$ & Hosseinzadeh, R. e cols. ${ }^{22}$ & Irã \\
\hline $\begin{array}{l}\text { A Physico-Chemical Study of the Cationic Surfactants Adsorption on } \\
\text { Montmorillonite }\end{array}$ & Praus, P. e Turicová, M. $^{23}$ & Tchecoslováquia \\
\hline Chemical Modifications of Nimesulide & Pericherla, S. e cols. ${ }^{24}$ & Índia \\
\hline $\begin{array}{l}\text { Electrochemical Fabrication of Sandwich Nanostructures Based on Anodic } \\
\text { Alumina }\end{array}$ & Li, Z. J. e Huang, K. L. ${ }^{25}$ & China \\
\hline Characterization by NMR of Ozonized Methyl Linoleate & Díaz, M. F. e Gavín, J. A. ${ }^{26}$ & Cuba/Espanhã \\
\hline Detection of Retinoic Acid Receptor Complex using Mass Spectrometry & Zhang, L. e Song, Z. ${ }^{27}$ & China \\
\hline $\begin{array}{l}\text { Synthesis and Antibacterial Activity of some Novel 2-Aroylimino-3-aryl-thiazolidin- } \\
\text { 4-ones }\end{array}$ & Saeed, A. e cols. ${ }^{28}$ & Paquistão/Alemanha \\
\hline $\begin{array}{l}\text { A New Sensitive Spectrophotometric Determination of Cypermethrin Insecticide } \\
\text { in Environmental and Biological Samples }\end{array}$ & Janghel, E. K. e cols. ${ }^{29}$ & Índia \\
\hline $\begin{array}{l}\text { Separation Study of Silver(I) Ion through a Bulk Liquid Membrane Containing } \\
\text { Meloxicam }\end{array}$ & Farhadi, K. e cols. ${ }^{30}$ & Irã \\
\hline $\begin{array}{l}\text { Nucleation and Growth Mechanism of Polycarbazole Deposited by } \\
\text { Electrochemistry }\end{array}$ & Abé, S. Y. e cols. ${ }^{31}$ & $\begin{array}{l}\text { França/Costa do } \\
\text { Marfim/Chile }\end{array}$ \\
\hline Design and Cytotoxic Evaluation of New Annonaceous Acetogenin Analogues & Krauss, J. e cols. ${ }^{32}$ & Alemanha \\
\hline Biotransformation of (S)-cis-verbenol with Nocardia corallina B-276 & Manjarrez, N. e cols. ${ }^{33}$ & México \\
\hline $\begin{array}{l}\text { Synthesis and Biological Activity of Allosteric Modulators of GABAB Receptors } \\
\text { Part 3. 3-(2,6-Bis-iso-propyl-4-hydroxyphenyl) propanols }\end{array}$ & Kerr, D. I. B. e cols. ${ }^{34}$ & Austrália/Irã \\
\hline $\begin{array}{l}\text { Potentiometric Coated Wire Electrode for Salicylate based on Zinc(II) } \\
\text { Acetylacetonate }\end{array}$ & Ardakani, M. M. e cols. ${ }^{35}$ & Irã \\
\hline $\begin{array}{l}\text { Enantioselective Transport of } R \text {-Clenbuterol through a Bulk Liquid Membrane } \\
\text { containing } O, O^{\prime} \text {-Dibenzoyl- }(2 S, 3 S) \text {-tartaric Acid }\end{array}$ & Jiao, F. e cols. ${ }^{36}$ & China \\
\hline $\begin{array}{l}\text { Poly(3-methylthiophene-co-3-octylthiophene) Based Solid-State Photoelectro- } \\
\text { chemical Device }\end{array}$ & Lemma, T. e Yohannes, T. ${ }^{37}$ & Etiópia \\
\hline $\begin{array}{l}\text { Synthesis and Nuclear Magnetic Resonance Shielding Effect of three Triazine-Linked } \\
\text { Porphyrin Compounds }\end{array}$ & Wang, K. e cols. ${ }^{38}$ & China \\
\hline
\end{tabular}


Tabela 1. continuação

Título
Synthesis and Characterization of ZnS Nanotubes with Crossed-channels
Screening Method for Rapid Determination of Polychlorinated Biphenyls in
Transformer Oil by Liquid-Liquid Extraction and Gas Chromatography-Mass
Spectrometry
Continuous Wavelet Transform and Chemometric Methods for Quantitative Resolution
of a Binary Mixture of Quinapril and Hydrochlorothiazide in Tablets
Complexes of Thallium(I) and Cadmium(II) with Dipeptides of L-phenylalanylglycine
and Glycyl-L-phenylalanine

Microwave-Assisted One-Pot Synthesis of Symmetrical 4H-Pyran-4-ones

Hydrogen Peroxide Assessment in Exhaled Breath Condensate: Condensing Equipment-Rapid Flow Injection Chemiluminescence Method

Microwave-Assisted Rapid and Regioselective Synthesis of $N$-(alkoxy-carbonylmethyl) Nucleobases in Water

Diosgenin Quantification by HPLC in a Dioscorea polygonoides Tuber Collection from Colombian Flora

Coumarins and Xanthones from the Seeds of Mammea siamensis

7,8 $\beta$-Dihydroponasterone A, a New Phytoecdysteroid from the Needles of the Japanese Yew, Taxus cuspidata

Clean-up of Extracts for Nitrated Derivatives of Polycyclic Aromatic Hydrocarbons Analyses prior to their Gas Chromatography Determination

Headspace Microextraction of Tin into an Aqueous Microdrop Containing Pd(II) and Tributyl Phosphate for its Determination by ETAAS

Studies of Electrochemical Behavior of SWNT-film Electrodes

Electrochemical Behaviour of Iron in Chlorinated Alkaline Media. The Effect of Slurries from Granite Processing

Synthesis, Herbicidal, Fungicidal and Insecticidal Evaluation of 3-(Dichlorophenyl)isocoumarins and ( \pm )-3-(Dichlorophenyl)-3,4-dihydroisocoumarins

Photoelectrochemical Cells based on Emeraldine Base Form of Polyaniline

$\mathrm{Fe}(\mathrm{III})$ Hepthyldithocarbamate as a New Collector for Flotation Separation and Preconcentration of $\mathrm{Cr}, \mathrm{Cu}$, and $\mathrm{Pb}$ from Fresh Waters before their Determination by ETAAS

Reaction of Aromatic Carboxylic Acids with Isocyanates using Ionic Liquids as Novel and Efficient Media

Synthesis of Macrocyclic Polyazomethines

Determination of Rhodium and Platinum by Electrothermal Atomic Absorption Spectrometry after Preconcentration with a Chelating Resin

A Novel and Highly Selective Conversion of Alcohols, Thiols, and Silyl Ethers to Azides using the 2,4,6-Trichloro[1,3,5]triazine/ $n$-Bu4NN3 System

C60-based Ebselen Derivative: Synthesis by Bingel Cyclopropanation and Enhanced Antioxidative and Neuroprotective Activity

Preparation and Characterization of Carboxyl-Group Functionalized Superparamagnetic Nanoparticles and the Potential for Bio-Applications

Determination of Trace Amounts of Copper in River and Sea Water Samples by Flame Atomic Absorption Spectrometry (FAAS) after Cloud-point Preconcentration

Determination of Thallium Traces by ETAAS after On-Line Matrix Separation and Preconcentration in a Flow Injection System

A Home-made Hybrid System for the Simultaneous Determination of Ergotamine, Dipyrone and Caffeine in Pharmaceutical Preparations

Failure of Montmorillonite K10 or Silica Gel to Promote the Conversion of Phenols to Quinones by Several Oxidants

Synthesis, Spectral Studies and Catalytic Activity of Ruthenium(II) Complexes with Organic Amide Ligands

$\begin{array}{cr}\text { Autores } & \text { País de orig } \\ \text { Chen, Y. e cols. }{ }^{39} & \text { China } \\ \text { Toledo, C. e cols. }^{40} & \text { Chile }\end{array}$

Dinç E. e Baleanu, D. ${ }^{41}$

Turquia/Romênia

Sharifi, S. e cols. ${ }^{42}$

Irã

Moghaddam, F. M. e cols. ${ }^{43}$

Irã

Vasiliou, E. G. e cols. ${ }^{44}$

Grécia

Qu, G. e cols. ${ }^{45}$

China

Niño, J. e cols. ${ }^{46}$

China

Laphookhieo, S. e cols. ${ }^{47}$

Tailândia

Shi, Q-W. e cols. ${ }^{48}$

China/Japão

Prycek, J. e cols. ${ }^{49}$

Tchecoslováquia

Hashemi, P. e cols. ${ }^{50}$

Irã

Rahman, M. M. e Jeon, I. C. ${ }^{51}$

Abreu, C. M. e cols. ${ }^{52}$

Coréia do Sul

Espanha

Qadeer, G. e cols. ${ }^{53}$

Paquistão/China

Sergawie, A. e cols. ${ }^{54}$

Áustria/Etiópia

Cundeva, K. e cols. ${ }^{55}$

República da

Macedônia

Irã

Mallakpour, S. e

Yousefian, H. ${ }^{56}$

Elizbarashvili, E. e cols. ${ }^{57}$

Geórgia

Rojas, F. S. e cols. ${ }^{58}$

Espanha

Akhlaghinia, B. e
Samiei, S.

Irã

Liu, X. e cols. ${ }^{60}$

China

Shan, Z. e cols. ${ }^{61}$

China

Goudarzi, N. ${ }^{62}$

Irã

Asadoulahi, T. e cols. ${ }^{63}$

Irã

Nezio, M. S. e cols. ${ }^{64}$

Argentina

Behrman, E. J. ${ }^{65}$

EUA

Ashok, M. e cols. ${ }^{66} \quad$ Índia 
Tabela 2. Artigos do exterior publicados em 2007 na Quim. Nova

\begin{tabular}{|c|c|c|}
\hline Título & Autores & País de origem \\
\hline $\begin{array}{l}\text { Preparation, Spectroscopic and Acidity Properties of two Hydrazones: An Organic } \\
\text { Lab Experiment }\end{array}$ & Rezende, M. C. e cols. ${ }^{67}$ & Chile \\
\hline Sobre a Primeira Lei da Termodinâmica. As Diferenciais do Calor e do Trabalho & Anacleto, J.; Anacleto, A. ${ }^{68}$ & Portugal \\
\hline Seeing Smells: Development of an Optoelectronic Nose & Suslick, K. S. e cols. ${ }^{69}$ & EUA \\
\hline \multicolumn{3}{|l|}{ Contenido de Flavonoides y Compuestos Fenólicos de Mieles Chilenas e Índice } \\
\hline Antioxidante & Muñoz, O. e cols. ${ }^{70}$ & Chile \\
\hline Metales Pesados y Toxicidad de Aguas del Río Aconcagua en Chile & Gaete, H. e cols. ${ }^{71}$ & Chile \\
\hline A Technique for High Recoveries from Vacuum Distillations & Langler, R. F. ${ }^{72}$ & Canadá \\
\hline The Interaction Between Sulfathiazole and Cobalt(ii): Potentiometric Studies & Bellú, S. e cols. ${ }^{73}$ & Argentina \\
\hline Sociedade Brasileira de Química - 30 Years On & Campbell, S. ${ }^{74}$ & Reino Unido \\
\hline $\begin{array}{l}\text { Assessment of Air Quality in Viana do Castelo, Portugal, in the Scope of the Polis } \\
\text { Programme }\end{array}$ & Alves, C. e Tomé, M.75 & Portugal \\
\hline $\begin{array}{l}\text { Analytical Investigation of Chromium and Zinc in Sweet, Sour and Bitter Tastingfruits, } \\
\text { Vegetables and Medicinal Plants }\end{array}$ & Tirmizi, S. A. e cols. ${ }^{76}$ & Paquistão \\
\hline $\begin{array}{l}\text { Efecto de la Naturaleza del Precursor sobre las Caracteristicas de las Nanoparticulas } \\
\text { de } \mathrm{SNO}_{2} \text { Sintetizadas }\end{array}$ & $\begin{array}{l}\text { Ararat-Ibarguen, C. E. } \\
\quad \text { e cols. }^{77}\end{array}$ & Colômbia \\
\hline $\begin{array}{l}\text { Estabilidad de la Glucosa Oxidasa en Sistemas Amorfos Formados por los Disacáridos } \\
\text { Sacarosa, Maltosa y Trehalosa }\end{array}$ & $\begin{array}{l}\text { Valenzuela, H. L. D. e } \\
\text { Ortiz, R. L. R. }{ }^{78}\end{array}$ & Colômbia/ Venezuela \\
\hline Inserção C-H de Carbenóides de Ródio em Água e Reutilização do Catalisador & Candeias, N. R. e cols. ${ }^{79}$ & Portugal \\
\hline $\begin{array}{l}\text { Size Distribution of Atmospheric Particulate Ionic Species at a Coastal Site in Por- } \\
\text { tugal }\end{array}$ & Alves, C. e cols. ${ }^{80}$ & Portugal \\
\hline $\begin{array}{l}\text { Desviaciones al Modelo Logarítmico-Lineal en la Solubilidad de Ibuprofén y Naproxén } \\
\text { en Mezclas Cosolventes Propilenoglicol-Agua }\end{array}$ & Vargas, E. F. e cols. ${ }^{81}$ & Colômbia \\
\hline $\begin{array}{l}\text { Un Método de Bajo Costo para la Determinación de Cobre a Nivel de Vestigios en } \\
\text { Matrices de Interés Ambiental por Espectrofotometría en Fase Sólida (EFS) }\end{array}$ & Pellerano, R. G. e cols. ${ }^{82}$ & Argentina \\
\hline
\end{tabular}

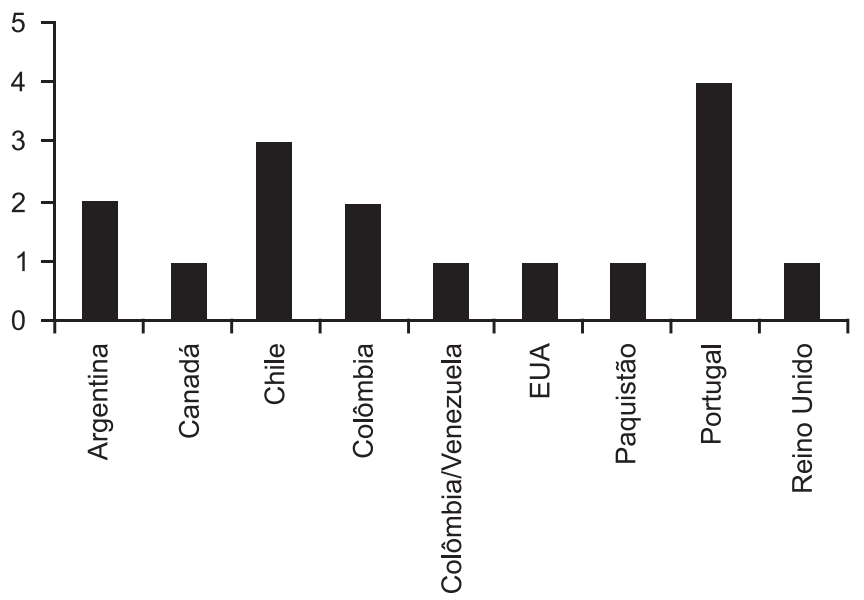

Figura 2. Publicações por país, em 2007, na Quim. Nova

\section{CONCLUSÃO}

Não há dúvida que o Fator de Impacto é um indicador importante, mas daí a ser usado como critério único em sistemas de avaliação vai uma distância muito grande. A Internacionalização da produção científica brasileira depende antes de tudo de revistas científicas brasileiras de qualidade e com capacidade para atrair artigos científicos de pesquisadores do exterior. Estas são duas das principais caracte- rísticas das revistas da Sociedade Brasileira de Química. O Journal of the Brazilian Chemical Society e a Química Nova são os principais cartões de visita da comunidade química brasileira. Foram apostas que deram certo e por isso devem ser prestigiadas pelos químicos brasileiros e pelas agências de fomento à pesquisa.

\section{AGRADECIMENTOS}

À FAPERJ, ao CNPq e à Dra E. Magalhães pelas informações fornecidas.

\section{REFERÊNCIAS}

1. Pinto, A. C.; de Andrade, J. B.; Quim. Nova 1999, 22, 448.

2. Strehl, L.; dos Santos, C. A.; Ciência Hoje 2002, 31, 34.

3. Kimura, E. T.; Arq. Bras. Endocrinol. Metab. 2008, 52, 925.

4. Hascall, V. C.; Bollen, J.; Hanson, R. W.; ASBMB Today 2007, July, 16.

5. Leta, J.; de Brito Cruz, C. H. Em Indicadores de Ciência, Tecnologia e Inovação no Brasil; Viotti, E. B.; Macedo, M. M., eds.; Editora UNICAMP: Campinas, 2003, p. 125.

6. Dupont, J.; Dias, L. C.; Quim. Nova 2008, 31, 1283.

7. de Torresi, S. I. C.; Pardini, V. L.; Dias, L. C.; Pinto, A. C.; de Andrade, J. B.; Magalhães, M. E. A.; Gil, P. E. de A.; Quim. Nova 2007, 30, 1491.

8. Marques, F.; Revista FAPESP 2008, Agosto, 34. 
9. Fong, S. S.; Seng, L.; Majri, N. B.; Mat, H. B.; J. Braz. Chem. Soc. 2007, $18,34$.

10. Fong, S. S.; Seng, L.; Mat, H. B.; J. Braz. Chem. Soc. 2007, 18, 41.

11. Pasha, C.; Narayana, B.; J. Braz. Chem. Soc. 2007, 18, 167.

12. Zamani, H. A.; Ganjali, M. R.; Adib, M.; J. Braz. Chem. Soc. 2007, 18, 215.

13. Norouzi, P.; Daneshgar, P.; Ganjali, M. R.; Moosavi-Movahedi, A.; J. Braz. Chem. Soc. 2007, 18, 231.

14. Khazaei, A.; Zolfigol, M. A.; Tanbakouchian, Z.; Shiri, M.; Rostami, A.; Iloukhani, H.; J. Braz. Chem. Soc. 2007, 18, 239.

15. Ghasemi, J.; Niazi, A.; Maeder, M.; J. Braz. Chem. Soc. 2007, 18, 267.

16. Varala, R.; Enugala, R.; Adapa, S. R.; J. Braz. Chem. Soc. 2007, 18, 291.

17. Mohsenzadeh, F.; Aghapoor, K.; Darabi, H. R.; J. Braz. Chem. Soc. 2007, 18, 297.

18. Matusiewicz, H.; Krawczyk, M.; J. Braz. Chem. Soc. 2007, 18, 304.

19. Patel, R. B.; Chikhalia, K. H.; Pannecouque, C.; Clercq, E.; J. Braz. Chem. Soc. 2007, 18, 312.

20. Tamrakar, U.; Pillai, A. K.; Gupta, V. K.; J. Braz. Chem. Soc. 2007, 18, 337.

21. Ganjali, M. R.; Rezapour, M.; Rasoolipour, S.; Norouzi, P.; Adib, M.; J. Braz. Chem. Soc. 2007, 18, 352.

22. Hosseinzadeh, R.; Bordbar, A-K.; Matin, A. A.; Maleki, R.; J. Braz. Chem. Soc. 2007, 18, 359.

23. Praus, P.; Turicová, M.; J. Braz. Chem. Soc. 2007, 18, 378.

24. Pericherla, S.; Mareddy, J.; Rani, G.; Gollapudi, P. V.; Pal, S.; J. Braz. Chem. Soc. 2007, 18, 384.

25. Li, Z. J.; Huang, K. L.; J. Braz. Chem. Soc. 2007, 18, 406.

26. Díaz, M. F.; Gavín, J. A.; J. Braz. Chem. Soc. 2007, 18, 513.

27. Zhang, L.; Song, Z.; J. Braz. Chem. Soc. 2007, 18, 526.

28. Saeed, A.; Abbas, N.; Flörke, U.; J. Braz. Chem. Soc. 2007, $18,559$.

29. Janghel, E. K.; Rai, J. K.; Rai, M. K.; Gupta, V. K.; J. Braz. Chem. Soc. 2007, 18, 590 .

30. Farhadi, K.; Bahar, S.; Maleki, R.; J. Braz. Chem. Soc. 2007, 18, 595.

31. Abé, S. Y.; Ugalde, L.; Valle, M. A.; Trégouët, Y.; Bernède, J. C.; J. Braz. Chem. Soc. 2007, 18, 601.

32. Krauss, J.; Bracher, F.; Synowitz, K.; Unterreitmeier, D.; J. Braz. Chem. Soc. 2007, 18, 691 .

33. Manjarrez, N.; Pérez, H. I.; Solís, A.; Luna, H.; Liévano, R.; Ramírez, M.; J. Braz. Chem. Soc. 2007, 18, 709.

34. Kerr, D. I. B.; Khalafy, J.; Ong, J.; Prager, R. H.; Rimaz, M.; J. Braz. Chem. Soc. 2007, 18, 721.

35. Ardakani, M. M.; Pourhakkak, P.; Salavati-Niasari, M.; J. Braz. Chem. Soc. 2007, 18, 782 .

36. Jiao, F.; Chen, X.; Hu, W.; Yang, L.; Huang, K.; J. Braz. Chem. Soc. 2007, 18, 804

37. Lemma, T.; Yohannes, T.; J. Braz. Chem. Soc. 2007, 18, 818.

38. Wang, K.; Fu, S.-T.; Zhang, Z.; Li, Z.-Y.; J. Braz. Chem. Soc. 2007, 18, 911.

39. Chen, Y.; Wu, Q.-S.; Ding, Y.-P.; J. Braz. Chem. Soc. 2007, 18, 924.

40. Toledo, C.; Valle, L.; Narváez, J.; Richter, P.; J. Braz. Chem. Soc. 2007, $18,937$.

41. Dinç, E.; Baleanu, D.; J. Braz. Chem. Soc. 2007, 18, 962.

42. Sharifi, S.; Nori-shargh, D.; Bahadory, A.; J. Braz. Chem. Soc. 2007, 18, 1011.

43. Moghaddam, F. M.; Bardajee, G. R.; Ismaili, H.; J. Braz. Chem. Soc. 2007, 18, 1024.

44. Vasiliou, E. G.; Makarovska, Y. M.; Pneumatikos, I. A.; Lolis, N. V.; Kalogeratos, E. A.; Papadakis, E. K.; Georgiou, C. A.; J. Braz. Chem. Soc. 2007, 18, 1040.

45. Qu, G.; Zhang, Z.; Guo, H.; Geng, M.; Xia, R.; J. Braz. Chem. Soc. 2007, 18, 1061 .

46. Niño, J.; Jiménez, D. A.; Mosquera, O. M.; Correa, Y. M.; J. Braz. Chem. Soc. 2007, 18, 1073.
47. Laphookhieo, S.; Promnart, P.; Syers, J. K.; Kanjana-Opas, A.; Ponglimanont, C.; Karalai, C.; J. Braz. Chem. Soc. 2007, 18, 1077.

48. Shi, Q.-W.; Dong, M.; Huo, C.-H.; Su, X.-H.; Li, X.; Yamada, T.; Kiyota, H.; J. Braz. Chem. Soc. 2007, 18, 1081.

49. Prycek, J.; Ciganekb, M.; Šimek, Z.; J. Braz. Chem. Soc. 2007, 18, 1125.

50. Hashemi, P.; Rahimi, A.; Ghiasvand, A. R.; Abolghasemi, M. M.; J. Braz. Chem. Soc. 2007, 18, 1145.

51. Rahman, M. M.; Jeon, I. C.; J. Braz. Chem. Soc. 2007, 18, 1150.

52. Abreu, C. M.; Covelo, A.; Díaz, B.; Freire, L.; Nóvoa, X. R.; Pérez, M. C.; J. Braz. Chem. Soc. 2007, 18, 1158.

53. Qadeer, G.; Rama, N. H.; Fan, Z.-J.; Liu, B.; Liu, X.-F.; J. Braz. Chem. Soc. 2007, 18, 1176.

54. Sergawie, A.; Yohannes, T.; Günes, S.; Neugebauer, H.; Sariciftci, N. Z.; J. Braz. Chem. Soc. 2007, 18, 1189.

55. Cundeva, K.; Pavlovska, G.; Stafilov, T.; J. Braz. Chem. Soc. 2007, 18, 1207

56. Mallakpour, S.; Yousefian, H.; J. Braz. Chem. Soc. 2007, 18, 1220.

57. Elizbarashvili, E.; Matitaishvili, T.; Topuria, K.; J. Braz. Chem. Soc. 2007, 18, 1254.

58. Rojas, F. S.; Ojeda, C. B.; Pavón, J. M. C.; J. Braz. Chem. Soc. 2007, 18, 1270

59. Akhlaghinia, B.; Samiei, S.; J. Braz. Chem. Soc. 2007, 18, 1311.

60. Liu, X.; Guan, W.; Ke, W.; J. Braz. Chem. Soc. 2007, 18, 1316.

61. Shan, Z.; Yang, W.-S.; Zhang, X.; Huang, Q. M. ; Ye, H.; J. Braz. Chem. Soc. 2007, 18, 1329.

62. Goudarzi, N.; J. Braz. Chem. Soc. 2007, 18, 1348

63. Asadoulahi, T.; Dadfarnia, S.; Shabani, A. M. H.; J. Braz. Chem. Soc. 2007, 18, 1353.

64. Nezio, M. S.; Pistonesi, M. F.; Centurión, M. E.; Palomeque, M. E.; Lista, A. G.; Band, B. S. F.; J. Braz. Chem. Soc. 2007, 18, 1439.

65. Behrman, E. J.; J. Braz. Chem. Soc. 2007, 18, 1461.

66. Ashok, M.; Prasad, A. V. S. S.; Ravinder, V.; J. Braz. Chem. Soc. 2007, $18,1492$.

67. Rezende, M. C.; Pizarro, C.; Millán, D.; Quim. Nova 2007, $30,229$.

68. Anacleto, J.; Anacleto, A.; Quim. Nova 2007, 30, 488.

69. Suslick, K. S.; Bailey, D. P.; Ingison, C. K.; Janzen, M.; Kosal, M. E.; McNamara III, W. B.; Rakow, N. A.; Sen, A.; Weaver, J. J.; Wilson, J. B.; Zhang, C.; Nakagaki, S.; Quim. Nova 2007, 30, 677.

70. Muñoz, O.; Copaja, S.; Speisky, H.; Peña, R. C.; Montenegro, G.; Quim. Nova 2007, 30, 848.

71. Gaete, H.; Aránguiz, F.; Cienfuegos, G.; Tejos, M.; Quim. Nova 2007, 30,885 .

72. Langler, R. F.; Quim. Nova 2007, 30, 1012.

73. Bellú, S.; Rizzotto, M.; Okulik, N.; Jubert, A.; Quim. Nova 2007, 30, 1136.

74. Campbell, S.; Quim. Nova 2007, 30, 1393.

75. Alves, C.; Tomé, M.; Quim. Nova 2007, 30, 1555.

76. Tirmizi, S. A.; Wattoo, M. H. S.; Mazhar, M.; Wattoo, F. H.; Memon, A. N.; Iqbal, J.; Quim. Nova 2007, 30, 1573.

77. Ararat-Ibarguen, C. E.; Montenegro, A.; Rodríguez-Páez, J. E.; Aragón, J. U.; Quim. Nova 2007, 30, 1578.

78. Valenzuela, H. L. D.; Ortíz, R. L. R.; Quim. Nova 2007, 30, 1633.

79. Candeias, N. R.; Gois, P. M. P.; Afonso, C. A. M.; Quim. Nova 2007, 30, 1772 .

80. Alves, C.; Pio, C.; Campos, E.; Barbedo, P.; Quim. Nova 2007, 30, 1938 .

81. Vargas, E. F.; Manrique, Y. J.; Pacheco, D. P.; Torres, N. S.; Martínez, F.; Quim. Nova 2007, 30, 1945.

82. Pellerano, R. G.; Romero, C. H.; Acevedo, H. A.; Vazquez, F. A.; Quim. Nova 2007, 30, 2020. 